\title{
Paediatric splenectomy: The Johannesburg experience
}

\author{
N Patel, ${ }^{1}$ BA Hons, MA, MB BCh; A Nicola, ${ }^{1}$ MBBCh; P Bennet, ${ }^{1}$ MB ChB; J Loveland, ${ }^{1}$ MB BCh, FCS (SA), Cert Paed Surg (SA); \\ E Mapunda, ${ }^{1}$ MB ChB, FCS (SA), Cert Paed Surg (SA); A Grieve, ${ }^{1}$ MB BCh, MMed (Surg), FC Paed Surg (SA)
}

${ }^{1}$ Department of Paediatric Surgery, University of the Witwatersrand, Johannesburg, South Africa

Corresponding author: N Patel (niravpatel44@gmail.com)

\begin{abstract}
Background. Splenectomy is an uncommon procedure in children, and data on children who underwent splenectomy in South Africa are sparse. Objective. To describe the profile, operative management and outcomes of children undergoing splenectomy.

Methods. The records for all children aged 0 to 16 years who underwent splenectomy at Charlotte Maxeke Johannesburg Academic (CMJAH) and Chris Hani Baragwanath Academic (CHBAH) hospitals between 2000 and 2015 were reviewed. Student's $t$-tests and $\chi^{2}$ tests were used to analyse the data.

Results. The mean age at surgery was 9.9 years (range 3-16). Most splenectomies $(91 \% ; n=30 / 33)$ were performed for haematological disorders and were open $(67 \% ; n=22 / 33)$. The mean post-operative length of stay (LOS) was shorter in the laparoscopic (4.5 days) than the open ( 7.1 days) groups $(p<0.05)$. Surgical complications were more common in the laparoscopic $(36 \%, 4 / 11)$ than open $(9 \% ; n=2 / 22)$ group, and in children older than the mean age at time of surgery. No cases of overwhelming post splenectomy infection (OPSI) were noted. At study completion, $61 \%(n=20 / 33)$ of patients were alive, $9 \%(n=3 / 33)$ had demised, and 30\% $(n=10 / 33)$ were lost to follow-up.

Conclusion. Local indications for paediatric splenectomy mirror those found in international literature. Mean and median postoperative lengths of stay (LOS) were shorter in the laparoscopic than open group, but relatively longer for both groups than reported internationally. Laparoscopy is not currently the preferred technique for splenectomy in our setting. All mortalities were due to progression of underlying disease and no cases of OPSI were recorded. The high loss-to-follow-up rate in this study is a significant barrier to accurate data collection, analysis and reporting.
\end{abstract}

S Afr J Child Health 2018;12(1):21-23. DOI:10.7196/SAJCH.2018.v12i1.1431

Splenectomy is a well-described procedure in children. As opposed to the adult literature, trauma is an uncommon indication for splenectomy in children. Frequently, failure of medical therapy to control the splenic sequelae of haematological disorders, e.g. splenomegaly, hypersplenism and massive infarction, necessitates splenectomy. ${ }^{[1-4]}$ Common examples of haematological disorders that necessitate splenectomy in children are hereditary spherocytosis (HS), sickle cell disease (SCD) and idiopathic thrombocytopaenic purpura (ITP). Although the pre-, peri- and postoperative management of paediatric patients undergoing elective and emergency splenectomy is well described within the international literature, there is a paucity of such literature in the South African (SA) context. ${ }^{[1,4]}$ The objective of our study was to describe the demographic profile, operative management and outcomes of children undergoing splenectomy in the largest academic paediatric surgical centres in South Africa between 2000 and 2015.

\section{Methods}

A retrospective record review of paediatric patients undergoing splenectomy at Chris Hani Baragwanath Academic Hospital (CHBAH) and Charlotte Maxeke Johannesburg Academic Hospital (CMJAH) between 2000 and 2015 was conducted. Ethics approval was obtained from the University of the Witwatersrand Human Research Ethics Committee (ref. no. M160643). All patients between the ages of 0 and 16 years who underwent splenectomy were included in the study. All candidates for inclusion were identified by means of the splenectomy specimens submitted to the National Health Laboratory Services (NHLS) during the study period. Demographic data, data on pre- and postoperative medical management, surgical technique, postoperative complications and postoperative length of hospital stay were collected. Descriptive statistics were performed using Microsoft Excel. Analytical statistics were performed using
Student's $t$-test and $\chi^{2}$ test. Complete records were retrieved for 33 of the 41 eligible patients.

\section{Results}

A total of 41 splenectomies were performed on children between January 2000 and December 2015. Complete records were found for 33 of the patients; $48 \%(n=16 / 33)$ patients undergoing splenectomy were male, while $52 \%(n=17 / 33)$ were female. The mean age at surgery was 9.9 years (median 9; range 3 - 16). The majority of the splenectomies $(91 \% ; n=30 / 3)$ were performed for haematological disorders and the remainder $(9 \% ; n=3 / 33)$ were performed due to malignancy or trauma. Table 1 describes the specific indications for surgery in our series; $67 \%(n=22 / 33)$ of splenectomies in our series were performed open, with $33 \%(n=11 / 33)$ performed laparoscopically. In a single case, laparoscopy was abandoned due to uncontrolled intra-abdominal haemorrhage. The mean postoperative hospital length of stay (LOS) was significantly shorter $(p<0.05)$ in patients who underwent laparoscopic splenectomy (4.5 days) than in those who had open surgery ( 7.1 days). The median postoperative

Table 1. Indications for surgery

\begin{tabular}{ll}
\hline Indication & $\boldsymbol{n}$ \\
\hline ITP & 13 \\
HS & 12 \\
SCD & 3 \\
CHA & 2 \\
Malignancy & 2 \\
Trauma & 1
\end{tabular}

HS = hereditary spherocytosis; ITP = idiopathic thrombocytopaenic purpura; $\mathrm{CHA}=$ congenital haemolytic anaemia; $\mathrm{SCD}=$ sickle cell disease. 
hospital LOS was also shorter in the laparoscopic (4.0 days) relative to the open (6.5 days) group. All patients in our series underwent total splenectomy. The incidence of postoperative complications was higher in the laparoscopic $(36 \% ; n=4 / 11)$ than the open group (9\%; $n=2 / 22)$. These data and the relevant complications are summarised in Table 2. All patients undergoing splenectomy received immunisations against Streptococcus pneumoniae, Haemophilus influenzae, and Meningococcus as per departmental protocol. Booster immunisations were given at follow-up where necessary. All patients received postoperative life-long antibiotic prophylaxis (Pen VK or erythromycin) as per the departmental protocol. At a two-year followup, 20 patients (61\%) were alive, $3(9 \%)$ had died, and $10(30 \%)$ had been lost to follow-up. These data are summarised in Table 3 .

\section{Discussion}

The role of the spleen has long been disputed. Splenomegaly was associated with poor health and poor athletic ability in the ancient Roman, Egyptian and Babylonian eras. ${ }^{[5]}$ Hippocrates considered the spleen the seat of black cholera and melancholy. ${ }^{[5]}$ It was this perception of splenomegaly as a source of physical and mental disability that led ancient physicians to seek out ways in which to remove the spleen or at least decrease its size. ${ }^{[5]}$ The first recorded splenectomy in Western medicine occurred in 1549, but it was not until the 19th century that elective splenectomies for splenomegaly were regularly performed. $^{[6]}$ Due to advances in anatomical knowledge and surgical technique, splenectomy became increasingly feasible. Fortunately, this improvement in surgical capability was accompanied by the knowledge that splenectomy performed for the wrong indication often had disastrous consequences. ${ }^{[5]}$

Currently, the most common indication for splenectomy in children is to treat the splenic effects of haematological conditions, e.g. hypersplenism, splenomegaly or splenic sequestration. ${ }^{[5]}$ The

Table 2. Complications of open and laparoscopic splenectomy $(n=6)$

\begin{tabular}{ll}
\hline & $n$ \\
\hline Open splenectomy $(n=2)$ & \\
Minor complications & 1 \\
$\quad$ Superficial wound sepsis & \\
Major complications & 1 \\
$\quad$ Right pneumothorax & \\
Laparoscopic splenectomy $(n=4)$ & \\
Major complications & 2 \\
$\quad$ Infected haematoma & 1 \\
$\quad$ Port-site hernia & 1 \\
Conversion to open surgery &
\end{tabular}

Table 3. Postoperative management and outcome

Candidates receiving antibiotic prophylaxis

Antibiotic prophylaxis

Penicillin VK

Erythromycin

Intermediate outcomes

Alive at follow-up 1 year post splenectomy

Alive at follow-up 2 years post splenectomy

Lost to follow-up at 1 year post splenectomy

Lost to follow-up 2 years post splenectomy

6

Deceased at 1 year post splenectomy

Deceased at 2 years post splenectomy first splenectomy for haematological disorders in children was performed in the early 20th century, more than 400 years after the first recorded splenectomy in adults. ${ }^{[5]}$ Notwithstanding significant improvements in the post-operative haematological profiles of these patients, splenectomised patients were also found to be especially prone to immediate and late postoperative sepsis. By the 1970s, this phenomenon and the associated role of encapsulated bacteria, such as $S$. pneumoniae, $H$. influenzae type B and Neisseria meningitidis types $\mathrm{A}$ and $\mathrm{C}$, had been labelled as overwhelming causes of postsplenectomy infection (OPSI). ${ }^{[7]}$ OPSI is a devastating consequence of splenectomy in children, with mortality rates of up to $50 \%$ in high-risk groups, i.e. young children, those with haematological disease and with malignancy. ${ }^{[8]}$ Although at highest risk within the first two years post-splenectomy, the risk of OPSI is lifelong. Appropriate pre- and postoperative management with vaccination, antibiotic prophylaxis and immediate management of suspected sepsis in splenectomised patients have enabled modern surgeons and physicians to safely perform splenectomies in children under the age of 4 in some centres. ${ }^{[7]}$

The indications for splenectomy in our series were similar to those found in the international literature, with the majority of cases performed for haematological disorders (namely ITP and HS). In total, 39\% $(n=13 / 33)$ of all cases were for ITP and 36\% $(n=12 / 33)$ for HS. Although laparoscopy is now the standard surgical approach for splenectomy in children, only $33 \%(n=11 / 33)$ of cases were performed laparoscopically in our series. ${ }^{[4,5,9]}$ In a single instance, open conversion was necessary due to excessive intra-abdominal haemorrhage. The high rate of open splenectomy in our series could possibly be due to the lack of appropriate equipment during the early years of the study, lack of the necessary laparoscopic skills, and the fact that patients who present with massive splenomegaly are not amenable to laparoscopy.

The complication rate for laparoscopic splenectomy in our series was $36 \%(n=4 / 11) ; 2$ patients developed infected haematomas, 1 had a port-site hernia, and 1 required open conversion due to haemorrhage. Both haematomas required open drainage and antibiotic treatment. The complication rate in our series of laparoscopic splenectomy contrasts with international rates of $1-5 \%,{ }^{[4,5]}$ and may be expected, given our relative inexperience with laparoscopic splenectomy in the early study period and the relatively low sample size. The complication rate for open splenectomy was $9 \%(n=2 / 22)$, with major complications accounting for $4.5 \%(n=1 / 22)$ and minor complications $4.5 \%(n=1 / 22)$ of the overall rate. The discrepancy in the complication rate for open splenectomy in our series and the rate of $1 \%$ reported in the international literature ${ }^{[4,5]}$ may again be due to the low sample size. Postoperative complications were more common in patients who were older than the mean age of 9.9 years $(n=16)-25 \%(n=4 / 16)$ of them suffered a significant postoperative complication. The complication rate was only $6 \%(n=1 / 17)$ in the 17 patients who were younger than the mean age of 9.9 years at the time of surgery. This finding may be due to numerous factors including the severity of the underlying disease process at the time of surgery and may suggest that earlier splenectomy may decrease the complication rate in our setting. Further study with a prospective and larger sample size is required in order to examine this relationship.

The mean postoperative LOS was significantly lower in the laparoscopic group (4.5 days) compared with the open group (7.1 days) $(p<0.05)$. Thus, our data reaffirm the conclusions of international literature with regards to the benefit of laparoscopic over open splenectomy in terms of postoperative LOS. ${ }^{[3,4,9-11]}$ This said, the postoperative LOS in both the open and laparoscopic groups was considerably longer in our series relative to international studies in which postoperative LOS ranges from $1.4-1.8$ days for laparoscopic splenectomy and 2.5 - 4.0 days for open splenectomy. ${ }^{[9,10]}$ In the local setting, access to quality healthcare is a significant 
concern and may prompt managing physicians to prolong hospital admission once patients are discharged from surgical care. This assertion is supported by the similar postoperative LOS for patients who experienced operative complications (mean 7.6 days) and those who did not (mean 6.2 days).

The mortality rate in our series was $9 \%(n=3 / 33)$ at 2 years post splenectomy. All mortalities were due to progression of underlying diseases, with one death due to progression of metastatic papillary serous adenocarcinoma of the ovary, another to an intracerebral bleed secondary to ITP and the last due to the cardiac sequelae of systemic lupus erythematosus. No case of OPSI was recorded. Mortality data and the incidence of OPSI in our setting are difficult to compare with the international literature due to the short follow-up period and the high number of patients who were lost to follow-up.

\section{Conclusion}

Indications for splenectomy were similar locally to those noted internationally, with haematological disorders accounting for the majority of cases. Laparoscopy, the standard surgical approach internationally, trails open surgery as the technique of choice in our setting. Postoperative lengths of hospital stay and complication rates (for both open and laparoscopic techniques) are longer and higher in our setting. This may be influenced by the late presentation of our patients, a skills shortfall in laparoscopic surgery of the spleen, prolonged post-surgical admission due to patient difficulties in reaccessing appropriate quality care once discharged from a tertiary centre, and the low sample size. Although no patients experienced OPSI in our series, the short follow-up period and high rate of loss to follow-up limit our ability to infer long-term outcomes in our patients. Further inquiry may centre on long-term patient outcomes, possible changes in surgical technique, given the knowledge and skills acquired in the past 15 years, and a comparison between the various academic paediatric surgical centres in South Africa.

Acknowledgements. The authors acknowledge the contributions of the Department of Paediatrics, Division of Haematology and Oncology, University of the Witwatersrand, for permitting access to their patient records.

Author contributions. NP, AN, PB, JL, EM and AG participated in concept/design, data analysis/interpretation, drafting and critical revision of the article, and data collection.
Funding. All research for this article was self-funded by the principal investigator and the Department of Paediatric Surgery, University of the Witwatersrand.

Conflicts of interest. None.

1. Al-Salem AH. Indications and complications of splenectomy for children with sickle cell disease. J Pediatr Surg 2006;41(11):1909-1915. https://doi. org/10.1016/j.jpedsurg.2006.06.020

2. Davies JM, Barnes R, Milligan D. Update of guidelines for the prevention and treatment of infection in patients with an absent or dysfunctional spleen. Clin Med 2002;2(5):440-443. https://doi.org/10.7861/clinmedicine.2-5-440

3. Luoto TT, Pakarinen MP, Koivusalo A. Long-term outcomes after pediatric splenectomy. Surgery 2015;159(6):1583-1590. https://doi.org/10.1016/j. surg.2015.12.014

4. Park A, Heinford BT, Hebra A, Fitzgerald P. Pediatric laparoscopic splenectomy. Surg Endosc 2000;(14):527-531. https://doi.org/10.1007/s004640000152

5. Wilkins BS. The spleen. Br J Haematol 2002;117:265-274. https://doi. org/10.1046/j.1365-2141.2002.03425.x

6. Sherman R. Perspectives in management of trauma to the spleen. J Trauma 1980;20(1):1-13. https://doi.org/10.1016/s0022-3468(80)80830-X

7. Lesher AP, Kalpatthi R, Glenn JB, Jackson SM, Hebra A. Outcome of splenectomy in children younger than 4 years with sickle cell disease. J Pediatr Surg 2009;44(6):1134-1138. https://doi.org/10.1016/j.jpedsurg.2009.02.016

8. Salvadori MI, Price VE. Preventing and treating infections in children with asplenia or hyposplenia. Paediatr Child Health 2014;19(5):271-274. https://doi. org/10.1093/pch/19.5.271

9. Rescorla FJ, Breitfeld PP, West KW, Williams D, Engum SA, Grosfeld JL. A case controlled comparison of open and laparoscopic splenectomy in children. Surgery 1998;124(4):670-676. https://doi.org/10.1067/msy.1998.91223

10. Minkes RK, Lagzdins M, Langer JC. Laparoscopic versus open splenectomy in children. J Pediatr Surg 2000;35(5):699-701. https://doi.org/10.1053/ jpsu.2000.6010

11. Qureshi FG, Ergun O, Sandulache VC, et al. Laparoscopic splenectomy in children. JSLS 2005;(9)4:389-392.

12. Eraklis AJ and Filler RM. Splenectomy in childhood: A review of 1413 cases. J Pediatr Surg 1972;7(4):382-388. https://doi.org/10.1016/0022-3468(72)90006-1

13. Bisharat N, Omari H, Lavi I, Raz R. Risk of infection and death among postsplenectomy patients. J Infect 2001;43(3):182-186. https://doi.org/10.1053/ jinf.2001.0904

14. Seims AD, Breckler FD, Hardacker KD, Rescorla FJ. Partial versus total splenectomy in children with hereditary spherocytosis. Surgery 2013;154(4):853-855. https://doi.org/10.1016/j.surg.2013.07.019

15. Rice HE, Oldham KT, Hillery CA, Skinner MA, O’Hara SM, Ware RE. Clinical and haematological benefits of partial splenectomy for congenital hemolytic anemias in children. Ann Surg 2003;237(2):281-288. https://doi.org/10.1097/01. sla.0000048453.61168.8f

Accepted 2 November 2017 